\title{
ON A GROUP THAT CANNOT BE THE GROUP OF A 2-KNOT 1
}

\author{
KUNIO MURASUGI
}

\begin{abstract}
It is proved that a homomorph of the group of trefoil knot cannot be the group of a 2-knot in 4-sphere.
\end{abstract}

In this short note we will give a negative answer to a problem proposed by Fox [2, Problem 28]. Fox asked if a finitely presented group that is a homomorph of a knot group and is infinite cyclic over its commutator subgroup is the group of a locally flat 2 -sphere in $R^{4}$. We prove

THEOREM. $G=\left(a, b: a^{2} b^{-3},[a, b]^{2}\right)$ cannot be the group of a locally flat $n$ sphere in $(n+2)$-sphere $S^{n+2}$ for $n \geqslant 2$, where $[a, b]=a b a^{-1} b^{-1}$.

Since $G / G^{\prime}$ is infinite cyclic and $G$ is a factor group of $G^{*}=\left(a, b: a^{2} b^{-3}\right)$ that is the group of the trefoil knot, our theorem gives a negative answer to the problem, where $G^{\prime}$ denotes the commutator subgroup of $G$.

Our proof is based on the following

Proposition 1 [4, p. 106]. If a finitely presented group $\pi$ is the group of a locally flat $n$-sphere $K^{n}$ in $S^{n+2}, n \geqslant 1$, i.e. $\pi \cong \pi_{1}\left(S^{n+2}-K^{n}\right)$, then

(1) $\pi / \pi^{\prime}$ is infinite cyclic,

(2) the weight of $\pi$ is one, and

(3) $H_{2}(\pi)=0$, where $H_{2}(\pi)$ denotes the second homology group of $\pi$ with integral coefficient and trivial action of $\pi$ on the coefficient group.

Furthermore, if $n \geqslant 3$, then (1), (2) and (3) are sufficient conditions for $\pi$ to be the group of some $n$-knot $K^{n}$ in $S^{n+2}$.

Our group $G$ satisfies (1) and (2), since $G^{*}$ does as the knot group. Therefore, to prove the theorem we must show

Proposition 2. $\mathrm{H}_{2}(G) \neq 0$.

Proof. Let $F$ be the free group with two free generators $a, b$. For a set, $X$, of elements of $F$, we denote by $X^{F}$ the normal closure of $X$ in $F$.

Now, let $R=\left\{a^{2} b^{-3},[a, b]^{2}\right\}^{F}$. Then $G \cong F / R$ and, by Hopf [3], $H_{2}(G)$ $=R \cap F^{\prime} /[R, F]$. Since $[a, b]^{2} \in F^{\prime}$, it suffices to show that $[a, b]^{2} \neq 1$ in the group

Received by the editors September 16, 1975.

AMS (MOS) subject classifications (1970). Primary 55A25.

Key words and phrases. $n$-knot, knot group.

${ }^{1}$ Research was supported by National Research Council grant A4034.

(c) American Mathematical Society 1977 


$$
H=\left(a, b:\left[a^{2} b^{-3}, a\right],\left[a^{2} b^{-3}, b\right],\left[[a, b]^{2}, a\right],\left[[a, b]^{2}, b\right]\right) .
$$

Let $S=\left\{a^{2} b a^{-2} b^{-1}, a b^{3} a^{-1} b^{-3},\left[[a, b]^{2}, a\right],\left[[a, b]^{2}, b\right]\right\} . \quad$ Obviously, $\quad H$ $\cong F / S^{F}$.

Consider the following sets.

$$
\begin{aligned}
T & =\left\{a^{2}, b^{3},[a, b]^{2}\left[b, a^{-1}\right]^{-2},[a, b]^{2}\left[b^{-1}, a\right]^{-2}\right\}, \\
U & =\left\{a^{2}, b^{3},(a b)^{3}(b a)^{-3}\right\}, \\
V & =\left\{a^{2}, b^{3},[a, b]^{2}\right\} .
\end{aligned}
$$

We claim now

LEMMA 1. $T^{F}=U^{F}$.

Proof. Probably the simplest way to prove this is to show that

(1) $(a b)^{3}(b a)^{-3}=1$ in $F / T^{F}$ and

(2) $[a, b]^{2}\left[b, a^{-1}\right]^{-2}=1,[a, b]^{2}\left[b^{-1}, a\right]^{-2}=1$ in $F / U^{F}$, and direct computation works as follows:

$$
\begin{aligned}
b^{-2} a b^{-1}\left\{(a b)^{3}(b a)^{-3}\right\} b a^{-1} b^{2} & =\left[b, a^{-1}\right]^{2}\left[a, b^{-1}\right]^{2} \\
& =[a, b]^{2}[a, b]^{-2}=1 \quad \text { in } F / T^{F},
\end{aligned}
$$

and

$$
\begin{aligned}
{[a, b]^{2}\left[b, a^{-1}\right]^{-2} } & =a b\left(a b^{-1} a b a\right) b^{-1}\left(a b a b^{-1} a\right) b a b^{-1} \\
& =a b\left(b a b a b^{-1} a b^{-1}\right) b^{-1}\left(b^{-1} a b^{-1} a b a b\right) b a b^{-1} \\
& =a b^{-1} a b a b a b a b^{-1} a b^{-1}=1 \quad \text { in } F / U^{F},
\end{aligned}
$$

and similarly,

$$
[a, b]^{2}\left[b^{-1}, a\right]^{-2}=1 \quad \text { in } F / U^{F} .
$$

Lemma 2. $(a b)^{3}(b a)^{-3} \in V^{F}$.

Proof. Again it is enough to show that $(a b)^{3}(b a)^{-3}=1$ in $F / V^{F}$. In fact,

$$
\begin{aligned}
(a b)^{3}(b a)^{-3} & =a b a b\left(a b a b^{-1}\right) a b^{-1} a b^{-1} \\
& =a b a b\left(b a b^{-1} a\right) a b^{-1} a b^{-1} \\
& =a b a b^{-1} a b a b^{-1}=1 \quad \text { in } F / V^{F} .
\end{aligned}
$$

Now we return to the proof of Proposition 2.

To lead a contradiction, we suppose $[a, b]^{2}=1$ in $H$. Then $[a, b]^{2}$ is contained in $S^{F}$ and hence $[a, b]^{2}$ is in $T^{F}$. By Lemma 1 , then, $[a, b]^{2}$ is in $U^{F}$. Lemma 2 now yields $U^{F}=V^{F}$. This implies that two groups $G_{1}=F / U^{F}$ and $G_{2}=F / V^{F}$ are isomorphic. It is impossible, however, because $G_{1}=$ 
$\left(a, b: a^{2}, b^{3},(a b)^{3}(b a)^{-3}\right)$ is denoted by $\langle 2,3 \mid 3\rangle$ in $[1$, p. 76$]$ and its order is 48 $\left[1\right.$, p. 77]. On the other hand, $G_{2}=\left(a, b: a^{2}, b^{3},[a, b]^{2}\right)$ has order $24[1, \mathrm{p}$. 134].

\section{REFERENCES}

1. H. S. M. Coxeter and W. O. J. Moser, Generators and relations for discrete groups, SpringerVerlag, Berlin, 1957. MR 19, 527.

2. R. H. Fox, Some problems in knot theory, Topology of 3-Manifolds and Related Topics, Prentice-Hall, Englewood Cliffs, N.J., 1962, pp. 168-176. MR 25 \#3523.

3. H. Hopf, Fundamentalgruppe und zweite Bettische Gruppe, Comment. Math. Helv. 14 (1942), 257-309. MR 3, 316.

4. M. A. Kervaire, On higher dimensional knots, Differential and Combinatorial Topology, Princeton Math. Ser., No. 27, Princeton Univ. Press, Princeton, N.J., 1965. MR 31 \# 2732.

Department of Mathematics, University of Toronto, Toronto, Ontario M5S 1A1, Canada 